\title{
The value of virtual natural history collections for botanical instruction in these times of the COVID-19 pandemic
}

\author{
J. Hugo Cota-Sánchez ${ }^{1}$ (1)
}

Received: 3 September 2020 / Revised: 22 September 2020 / Accepted: 26 September 2020 / Published online: 19 October 2020

(c) Botanical Society of Sao Paulo 2020

With the return to classes, the unprecedented circumstances of the COVID-19 pandemic have created diverse challenges for faculty and students in all areas of education (Baldini 2020; Cota-Sánchez 2020). Consequently, the world teaching scenarios have been enhanced with health and safety measures to re-open schools during the pandemic. In this Letter, I discuss the impact of COVID-19 on the curriculum of biological sciences with an emphasis on courses in plant (botany-related) sciences. Based on my own experience, I consider some options on how to effectively deliver remote courses and train students in botany, taxonomy, and plant systematics using virtual collections and, whenever possible, fresh specimens.

As a result of the pandemic, universities around the world have moved to a hybrid of in-person and online courses. At the University of Saskatchewan (USASK), a few face-toface courses with a limited number of students are being offered under strict safeguarding protocols. As a professor of botany, I am heading into my fourth week of teaching Biol. 323-Plant Systematics and Evolution online, a course with extensive coverage about the taxonomy, phylogeny, and evolution of vascular plants. This class provides students with identification skills focused on local flora, but examples and taxonomic keys from other world regions are used. Thus far, my remote teaching experience has been quite rewarding and satisfying. At first, I found it rather daunting because all the computer accessories and applications involved, but I quickly adapted, and nowadays the work has become more and more enjoyable and comfortable with the use of live and digital resources as supporting teaching material, as explained next.

$\triangle$ J. Hugo Cota-Sánchez

hugo.cota@usask.ca

1 Department of Biology and W.P. Fraser Herbarium, University of Saskatchewan, 112, Science Place, Saskatoon, SK S7N 5E2, Canada
Foremost, contrasting with the traditional face-to-face teaching using diverse representative plant material, the online environment doesn't seem to translate very well when it comes to delivering technical botanical terminology and the associated plant structures without actually giving students the opportunity to manually dissect and examine a specimen. It is extremely difficult to have a remote class in which all students have access to the same plant material; hence, the effectiveness of knowledge transfer may be affected. In my experience, face-to-face guided, hands-on training to efficiently dissect and observe plant parts and structures is significant, and perhaps a must, for students to master plant identification skills.

With the shift to online classes, botany and plant systematics instructors, including me, have started using virtual herbaria more heavily. The vast number of digital archives available worldwide is an important factor to make remote teaching effective. Herbaria have been, are, and will continue to be the main learning centers for plant taxonomy, systematics, biodiversity, and management of natural history collections (Cota-Sánchez and Harms 2009). These institutions are training hubs of highly qualified personnel and new generations of the keepers of the world's plant diversity (Smith and Figueiredo 2009). Within this context, the significance of historical and modern botanical collections can be integrated into online education (Pantalony 2020). A wide array of teaching assignments (individual or team) can be made available in the form of quizzes for plant parts, family descriptors, monographs, phylogenetic studies, or digitization of herbarium specimens to identify biodiversity and endemic rich-species areas (Neto et al. 2016; Ostroski et al. 2020), among other possibilities. Also, because historical collections house abundant information not illustrated in texts or on the Internet, I have been using them to compare old and recently collected specimens of the same species to discuss the value of type specimens, botanical collectors, and issues of nomenclature. 
I also rely on my own institution, the W.P. Fraser Herbarium (SASK), to support my remote teaching. SASK has a digital database relevant to taxonomy, biodiversity, and conservation in the province of Saskatchewan. Concise information documents the province's past and present plant diversity, which along with preceding and present botanical explorations has enhanced the knowledge of the region's plant diversity. A type collection (Cota-Sánchez et al. 2004) and a compilation of carnivorous plant specimens (Baalim et al. 2013) are also available. Other online teaching resources include taxonomic keys and digital images of representative Saskatchewan plant families and species available at the Virtual Herbarium (https://www.usask.ca/biolo gy/virtualherbarium/) and the Virtual Herbarium of Plants at Risk in Saskatchewan (https://www.usask.ca/biology/ rareplants_sk/root/htm/en/researcher/index.php). Additionally, I use proactive approaches, such as immersing students in hands-on and fun culinary activities in my botany classrooms. My teaching methods entice students with demos using live plants and plant-based food in lectures. This scheme allows scholars to learn in an enjoyable environment the taxonomy and plant parts eaten, e.g., fruit, seeds, leaves, roots, etc. However, the delivery has not been as effective as I expected. Despite the additional camera gadgets I have for the show and tell session, students complain about the limited screen view, size, and resolution of plant structures being discussed; hence, face-to-face training is needed.

In contrast, computer technology has allowed vast progress in the digitization of botanical collections. The COVID-19 crisis is a prospect to exploit these precious resources as part of the tools for online teaching. In addition to botany, taxonomy, and field botany, these resources can be adapted to numerous classes, such as plant ecology, ethnobotany, agriculture, dendrology, forestry, etc. I am confident that we can educate and train the next generation of taxonomists using the wealth of information stored in virtual herbaria. The possibilities are endless, but due to the short nature of this letter, I can't give specific examples. A key factor is transitioning and adapting the online resources to the teaching goals. Nonetheless, even with the amazing, welldigitized images available virtually worldwide and the different camera resources for online education, teaching plant identification and plant structures has been a challenging, yet fun (sometimes frustrating) endeavor requiring a great deal of creativity and patience.

In conclusion, all fields of biology, arts, social sciences and beyond should expand and benefit from the use of virtual collections in education. Personally, even with the existing pressure and preparation time for lectures and the challenges to effectively use live specimens, I look forward to each of my classes because I love the interaction with students and the opportunity to grow as an educator and mentor for future botanists. I continue to enjoy the challenge that COVID-19 has brought to education pushing us outside the traditional teaching arena. I am optimistic of the endless possibilities for distance learning using virtual collections and exhibits, such as those available at JSTOR Global Plants (https ://plants.jstor.org), the C.V. Starr Virtual Herbarium, New York Botanical Garden (https://sweetgum.nybg.org/science/ $\mathrm{vh} /$ ), the Species Link Network (https://www.splink.org.br), the Plant List (https://www.theplantlist.org), to name a few. The new remote educational atmospheres can potentially be made available to all people regardless of location or resources as long as there is a computer, Internet access, enthusiasm, and motivation for learning.

\section{References}

Baalim F, Peters C, Cota-Sánchez JH (2013) Distribution and natural history of carnivorous plants of Saskatchewan, Canada. Check List 9:883-893

Baldini RM (2020) The impact of COVID-19 crisis on Plant Taxonomy: will we be able to approach to plant taxonomy as in the past? Webbia 75:3-4

Cota-Sánchez JH (2020) In Press) A complementary note to Baldini's article "The impact of COVID-19 crisis on Plant Taxonomy: will we be able to approach to plant taxonomy as in the past?". Webbia 75:xx

Cota-Sánchez JH, Harms VL (2009) The W.P. Fraser Herbarium (SASK) of the University of Saskatchewan: past, present and future. Blue Jay 67:97-104

Cota-Sánchez JH, Olson RW, Haraldson J (2004) The type collection of the W.P. Fraser (SASK) herbarium of the University of Saskatchewan. Polibotánica 17:131-138

Neto LM, Furtado SG, Zappi DC, de Oliveira Filho AT, Forzza RC (2016) Biogeography of epiphytic angiosperms in the Brazilian Atlantic forest, a world biodiversity hotspot. Braz J Bot 39:261-273

Ostroski P, Saiter FZ, Amorim AM, Fiaschi P (2020) Angiosperm endemism in a Brazilian Atlantic Forest biodiversity hot-point. Braz J Bot 43:397-404

Pantalony D (2020) What remains: the enduring value of museum collections in the digital age. HoST-J Hist Sci Technol 14:160-182

Smith GF, Figueiredo E (2009) Capacity building in taxonomy and systematics. Taxon 58:697-699

Publisher's Note Springer Nature remains neutral with regard to jurisdictional claims in published maps and institutional affiliations. 